\title{
Evaluation of the Pontine Perforators of the Basilar Artery Using Digital Subtraction Angiography in High Resolution and 3D Rotation Technique
}

\author{
S. Lescher, T. Samaan, and J. Berkefeld
}

\begin{abstract}
BACKGROUND AND PURPOSE: Compromise of perforating branches of the basilar artery resulting in brain stem infarctions has been described as a major complication of intracranial stent placement for basilar artery stenosis or after implantation of endovascular flow diverters. Descriptions of pontine arteries are mainly based on examinations of injection specimens; however, there is a lack of consistent presentation of the small branches of the basilar artery in the imaging literature. Therefore, we retrospectively analyzed DSA images and $3 \mathrm{D}$ rotational angiography with a review of literature for an imaging definition of microvascular anatomy of the brain stem.
\end{abstract}

MATERIALS AND METHODS: We retrospectively analyzed 2k DSA images (detector format $32 \times 32 \mathrm{~cm}$; image matrix $2480 \times 1920$ pixels) and 3D rotational angiography reconstructions ( 5 second DSA, subtraction technique) obtained on Axiom Artis zee biplane neuroradiologic angiography equipment using standard protocol.

RESULTS: On 2D and 3D DSA images, small arterial side branches of the basilar artery can be demonstrated in each of the cases but with a wide variation in the visibility of these vessels. Compared with 2D DSA images, 3D DSA reconstructions allow superior visualization of the small branches of the basilar artery.

CONCLUSIONS: Our results demonstrate that 2D DSA and 3D-rotation techniques are able to reliably visualize the penetrating branches of the brain stem in vivo. No zone of basilar artery is free from important side branches. Collateral pathways between circumferential and perforating arteries are occasionally detectable. In the future, further refinement of imaging techniques is necessary to increase the reliability of small vessel angiography to use this data for risk assessment before stent placement and aneurysm treatment.

ABBREVIATIONS: 3DRA $=3$ D rotational angiography; $2 \mathrm{k}$ matrix DSA $=$ detector format $32 \times 32 \mathrm{~cm}$; image matrix $2480 \times 1920$ pixels

C ompromise of perforating branches of the basilar artery has been described as a major complication of intracranial angioplasty and stent placement or implantation of endovascular flow diverters. ${ }^{1,2}$ Because of the small diameter of these side branches of $383 \mu \mathrm{m}$, visualization by different means of angiographic imaging like CT- or MR-based angiography is difficult and uncertain. ${ }^{3}$

High-resolution DSA images with $2 \mathrm{k}$ matrix (detector format $32 \times 32 \mathrm{~cm}$; image matrix $2480 \times 1920$ pixels) and especially flat detector CT with pixel sizes of $308 \mu \mathrm{m} \times 308 \mu \mathrm{m}(2 \times 2$ binning $)$, using a reconstructed section thickness of $0.1 \mathrm{~mm}$ and highcontrast resolution of $\mathrm{CT}$ and $3 \mathrm{D}$ reconstructions, provide a new

Received November 3, 2013; accepted after revision February 6, 2014.

From the Institute of Neuroradiology, Hospital of Goethe University, Frankfurt am Main, Germany.

Please address correspondence to Stephanie Lescher, MD, Institute of Neuroradiology, Hospital of Goethe University, Schleusenweg 2-16, 60528 Frankfurt am Main, Germany; e-mail: stephanie.lescher@kgu.de

- Indicates open access to non-subscribers at www.ajnr.org

http://dx.doi.org/10.3174/ajnr.A3981 basis to display these small vessels on recent flat detector neuroangiography systems. ${ }^{4}$ We hypothesized that the 3D-rotation technique allows superior visualization of the small branches of the basilar artery than 2D DSA images do.

The microvascular anatomy of the vertebrobasilar junction and the distal basilar tip has been described in detail in the neurosurgical and neuroradiologic literature. ${ }^{5-7}$ Remarkably, there are no comparable descriptions of the proximal and middle portion of the basilar artery in the imaging and neurosurgical literature. As such, anatomic knowledge is based on examinations of post mortem injection preparations., ${ }^{8,9}$

A continuation of patterns of the spinal cord vascular supply has been postulated for the brain stem with additional circumferential branches for the pons and cerebellum. ${ }^{7}$

The presence of perforating branches is known; however, in the past, angiographic imaging was far away from reliable pattern recognition and display of potential collateral pathways.

The lack of consistent descriptions in the imaging literature raised the question of whether the latest generations of flat detec- 
tor neuroangiography allow for more reliable visualization of the pontine branches of the basilar artery with the use of $2 \mathrm{k}$ matrix DSA images and 3D rotational angiography.

Therefore, we retrospectively analyzed $2 \mathrm{k}$ DSA images and 3D rotational angiography reconstructions together with a review of the literature for an imaging definition of the microvascular anatomy.

\section{MATERIALS AND METHODS}

For retrospective analysis, we identified 41 consecutive cases with indications for selective vertebral angiography and 3D rotational angiography (3DRA) in a period from January 2011 until March 2013. Twenty-one datasets with minor image quality due to incomplete contrast filling or movement artifacts were excluded. Twenty datasets with adequate image quality were selected for further evaluation.

The protocol was authorized by the ethics committee of Frankfurt.

All angiograms and 3DRA data were obtained on an Axiom Artis zee biplane (Siemens, Erlangen, Germany) neuroradiologic angiography system. The angiographic system is equipped with a flat panel detector with $2480 \times 1920$ pixels ( $2 \mathrm{k}$ matrix) and a pixel pitch of $154 \mu \mathrm{m} \times 154 \mu \mathrm{m}$.

For the evaluation of pontine arteries, 2k DSA images using high-resolution technique and 3DRA runs of the basilar artery between the vertebrobasilar and the pontomesencephal junction were analyzed. Indications for DSA and especially 3DRA were justified by suspected pathologies like aneurysms (ruptured/unruptured), arteriovenous malformations, stenosis, infarctions, or vasculitis.

\section{DSA with 2k Matrix}

Compared to former angiographic systems equipped with an $1 \mathrm{k}$ image matrix of $1240 \times 960$ pixels, the newest generations of angiographic systems provide improved and higher spatial resolution using a $2 \mathrm{k}$ image matrix of $2480 \times 1920$ pixels and a flat detector zoom format of $32 \times 32 \mathrm{~cm}$ in each plane. For retrospective evaluation of DSA images in standard Towne and lateral projections, we selected 20 consecutive cases with normal findings in the intracranial vertebrobasilar circulation and good image quality. DSA images were obtained using a standard program with a variable frame rate with 4 frames per second in the arterial phase, a small focus with a focal spot size of $0.3 \mathrm{~mm}$, and edge enhancement reconstruction algorithm. A flat detector zoom format of $32 \times 32 \mathrm{~cm}$ in each plane was used. In addition, $4-6 \mathrm{~mL}$ of non-ionic contrast material was applied via hand injection after selective catheterization of the dominant vertebral artery. The average effective dose of this examination was $2.7 \mathrm{mSv}$ measured in an anthropomorphic Alderson-Rando phantom with thermoluminescence dosimetry.

Image evaluation was done retrospectively by consensus of 2 experienced reviewers who counted the number of visible side branches at each side of the basilar artery and distinguished between circumferential arteries and AICA variants. They described whether circumferential arteries were displayed in a segmental or more irregular network pattern and whether common trunks or anastomoses between adjacent branches were present.

The reviewers also looked for direct paramedian pontine perforators.

\section{D Rotation Technique}

In the 20 patients with indications for 3DRA of the posterior circulation, we reconstructed the stored raw data of 3D DSA runs with a scan time of 5 seconds. A native rotational run was followed by contrast-enhanced images after mechanical intra-arterial injection of $20 \mathrm{~mL}$ of nonionic contrast material (Ultravist 240; Bayer-Schering, Berlin, Germany) into the dominant vertebral artery with a flow of $3 \mathrm{~mL} / \mathrm{s}$. Injection was started 2 seconds before the contrast-enhanced run. In each run, 133 images were obtained with a scan time of 5 seconds. The average effective dose of this examination was $0.9 \mathrm{mSv}$ measured in an anthropomorphic Alderson-Rando phantom with thermoluminescence dosimetry. The spatial resolution of flat detector CT was reported to be 3.0 $\mathrm{lp} / \mathrm{mm}$ with no binning and $1.5 \mathrm{lp} / \mathrm{mm}$ with $2 \times 2$ binning for high-resolution modes., ${ }^{40}$

The 3DRA raw data were transferred to a dedicated angiographic workstation (Siemens). The 3D datasets and CT-like images of subtraction angiograms were reconstructed using standard volume rendering and flat detector CT algorithms (DynaCT-arterial). MPR with section thicknesses of $0.1 \mathrm{~mm}$ and MIP with section thicknesses between 6 and $10 \mathrm{~mm}$ as well as volume rendering reconstructions with bicolored window and threshold settings were used for analysis of small vessels.

Datasets with a basilar artery stenosis and a midbrain AVM were analyzed as illustrative cases and additional proof of the value of our technique.

The number and pattern of pontine arteries was determined retrospectively by consensus of 2 reviewers. Analog to $2 \mathrm{k}$ DSA evaluation, the 2 reviewers counted the number of visible side branches at each side of the basilar artery and looked for direct paramedian midline perforators to determine origin and course of the small brain stem vessels. Especially in pathologic cases with stenotic lesion, we looked for potential collaterals between the small brain stem vessels according to rotating reconstructions. Perforators originating directly from the basilar artery were evaluated according to subtracted flat detector CT images.

\section{RESULTS}

\section{Biplane 2k DSA Images in Standard Projections}

Circumferential pontine arteries can be regularly demonstrated on DSA images of the basilar artery obtained with recent technology. The most common pattern is a segmental pattern of up to 10 circumferential arteries with slightly descending course around the pons. The origins from the dorsal surface of the basilar artery are best seen on lateral projection in an average distance of 1.5-3.5 $\mathrm{mm}$ between 2 "segmental" vessels and some variations between left and right. Common trunks bearing origins of several arteries, longitudinal anastomoses between different arteries, or midline perforators to the brain stem are occasionally seen.

\section{D and Flat Panel CT Angiography Reconstructions}

Compared with DSA images, 3DRA and flat detector CT reconstructions are superior in demonstrating anatomic patterns of circumferential arteries and direct pontine perforators (Table).

The basic patterns derived from the analysis of 20 cases are up to 10 circumferential arteries on each side with a characteristic downward-curving route around the pons (Fig 1), which can be 
Basic patterns of the small side branches of the basilar artery in 2k DSA and 3DRA imaging studies

\begin{tabular}{|c|c|c|c|c|c|c|c|c|c|c|c|c|}
\hline & \multicolumn{3}{|c|}{$\begin{array}{c}\text { Number of Segmental } \\
\text { Arteries Right }\end{array}$} & \multicolumn{3}{|c|}{$\begin{array}{c}\text { Number of Segmental } \\
\text { Arteries Left }\end{array}$} & \multicolumn{3}{|c|}{$\begin{array}{l}\text { Direct Pontine } \\
\text { Perforators }\end{array}$} & \multirow[b]{2}{*}{ Collaterals ${ }^{\mathrm{a}}$} & \multirow{2}{*}{$\begin{array}{c}\text { Common Trunks of } \\
\text { Circumferential } \\
\text { Arteries }\end{array}$} & \multirow{2}{*}{$\begin{array}{c}\text { Pial } \\
\text { Network }\end{array}$} \\
\hline & $0-3$ & $4-7$ & $8-10$ & $0-3$ & $4-7$ & $8-10$ & Caudal & Medial & Rostral & & & \\
\hline $2 \mathrm{k}$ DSA & 10 & 7 & 3 & 5 & 11 & 4 & 0 & 0 & 0 & 6 & 8 & 1 \\
\hline 3DRA & 6 & 13 & 1 & 5 & 13 & 2 & 20 & 20 & 20 & 11 & 10 & 6 \\
\hline
\end{tabular}

${ }^{a}$ Collaterals are defined as small anastomosis with a descending course connecting 2 adjacent circumferential arteries.

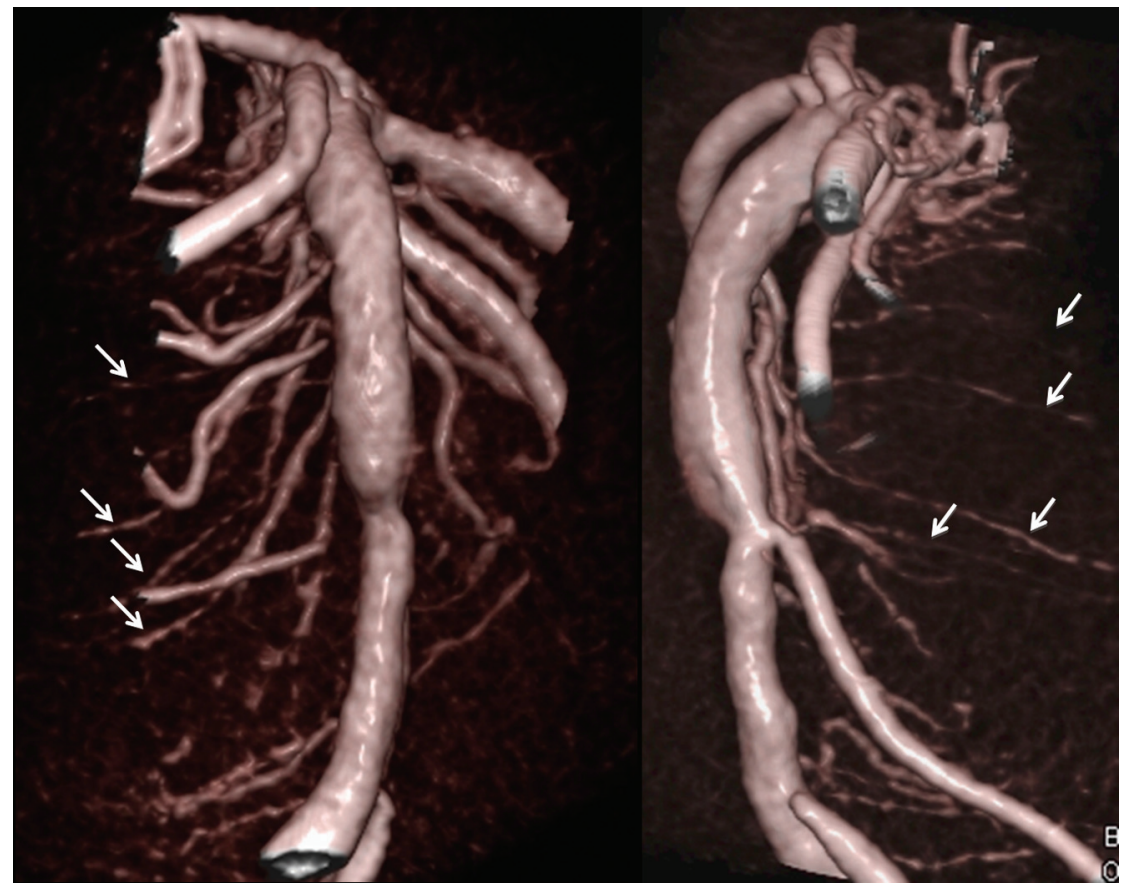

FIG 1. Segmental pattern and descending course of circumferential pontine arteries (arrows) on volume rendering reconstructions of a DynaCT dataset of the basilar artery in Towne and lateral projection.
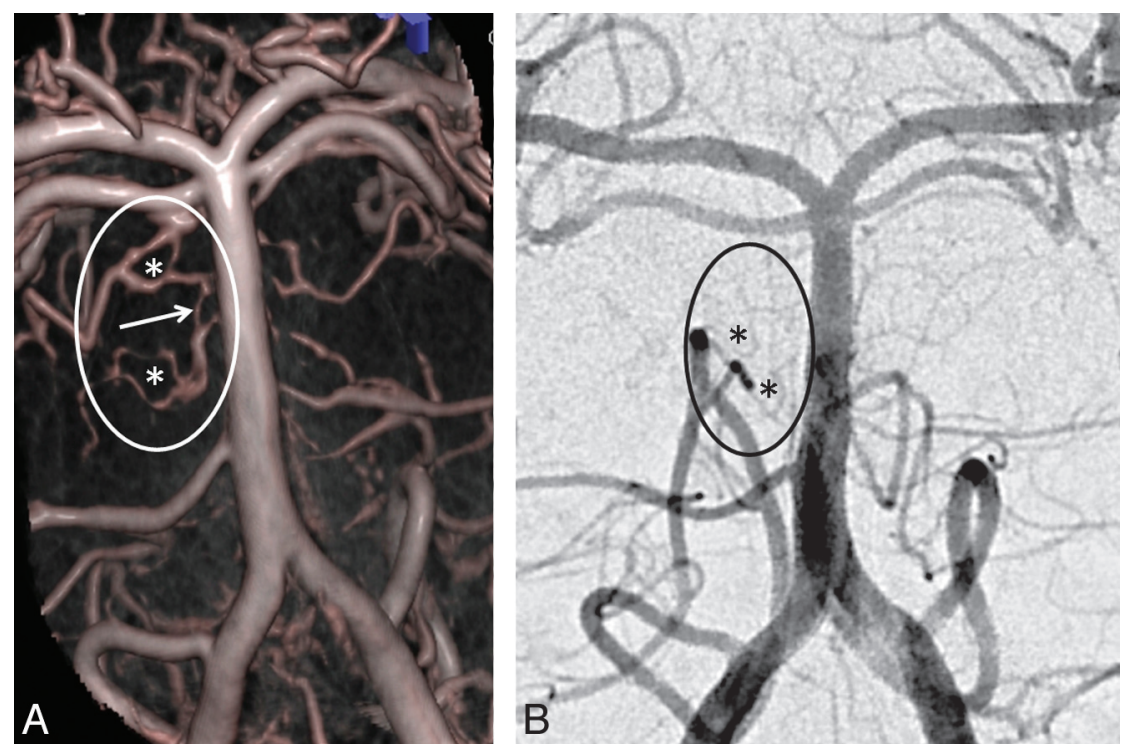

FIG 2. Another case shows a less regular pial network pattern $\left(^{*}\right)$ with connections (arrow) between adjacent arteries that are better demonstrated on DynaCT reconstructions $(A)$, than on high resolution DSA with $2 \mathrm{k}$ matrix $(B)$.

visualized more clearly in 3DRA compared with $2 \mathrm{k}$ DSA images. The number of the segmental arteries on each side of the basilar artery is the same in both 3DRA and 2k DSA images (Table). The distance between the origins of the circumferential branches ranged from 2.6$4.0 \mathrm{~mm}$. Transversal flat detector CT reconstructions (DynaCT arterial mode) show that the circumferential arteries during their downward-curving route toward the tegmentum of the pons give off peripheral perforating branches extending to the pontine parenchyma. Asymmetries between left and right and variations in size have been revealed as common variation.

In 10 of 20 cases, several circumferential arteries arose from a common trunk (Table).

Descending course and tortuosities of circumferential arteries may mimic longitudinal anastomoses or arcade-like connections that are truly present in 6 cases with a more pial-like network and in a further 11 cases with longitudinal anastomoses between adjacent circumferential arteries close to the origin and in no cases in the dorsolateral periphery of the brain stem (Table; Fig 2). 3DRA dataset reconstructions are able to differentiate between true anastomosis and vessel overlap.

Analogous to the sulco-comissural arteries in the spinal cord ${ }^{7,8}$ direct perforators to the pons could be identified as small paired vessels arising from the dorsal surface of the basilar artery and extending into the parenchyma. Direct pontine perforators could be identified on 3 levels (Fig 3): at the pontomesencephal junction (rostral), midbasilar (central), and vertebrobasilar junction (caudal). These 3 groups could be defined in all the evaluated images.

The AICA as the main branch of the midbasilar artery was seen in all cases. A large caliber of the AICA seems to be associated with smaller and less numerous other circumferential arteries.

\section{Cases with Pathologies}

In 2 cases with hemodynamically relevant stenosis of the basilar artery and the intradural part of the vertebral artery (V4), we identified small collaterals connecting circumferential arteries of the basilar artery bridging the stenosis (Fig 4). 

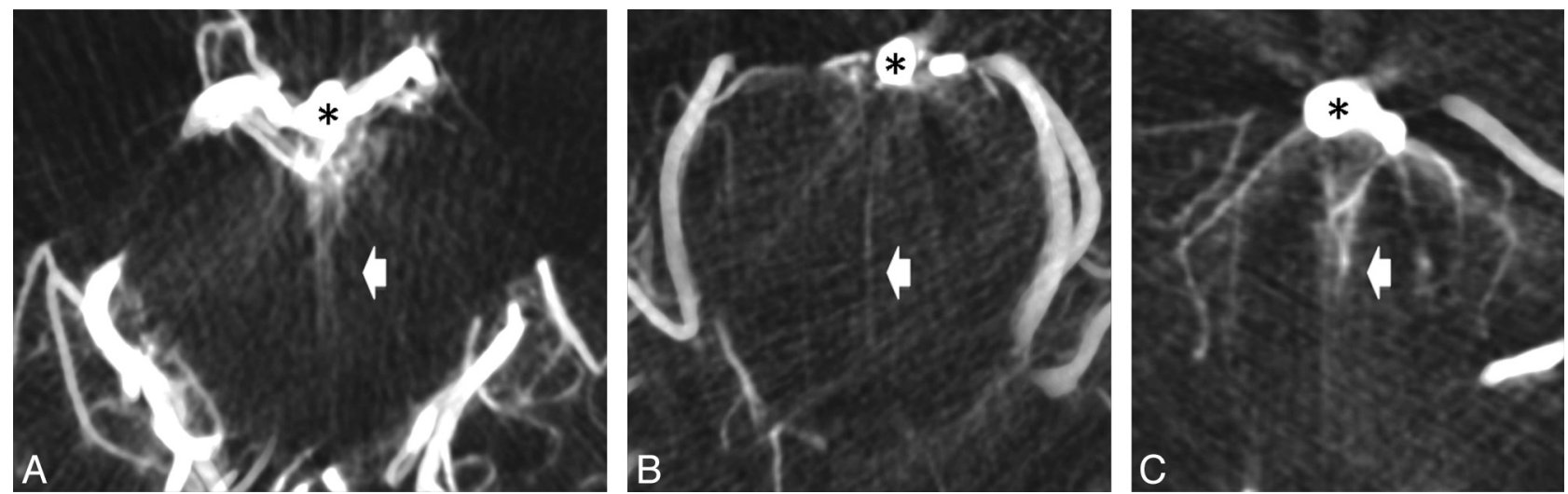

FIG 3. Axial cuts of DynaCT dataset with thin sections of $3 \mathrm{~mm}$ reconstructed from 3D rotational angiogram shows ventral pontine perforators (bright arrows) originating from the basilar artery $\left(^{\star}\right)$ at the junction between mesencephalon and pons $(A)$, at the midpontine level $(B)$, and at the vertebrobasilar junction (C).
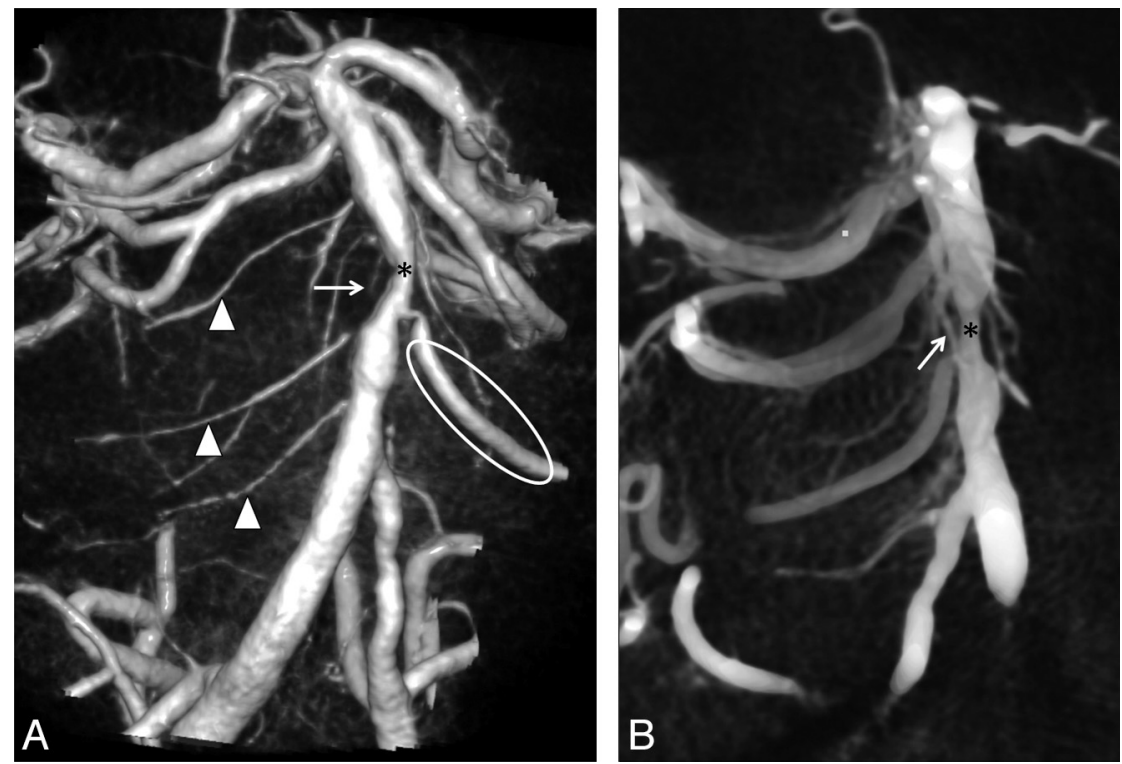

FIG 4. $A$, Volume rendering reconstructions of $3 D$ rotational angiogram show a segmental pattern with several circumferential pontine arteries on the right side (arrowheads) and a dominant AICA (ellipse) and less other pontine branches on the left side in a patient with a midbasilar stenosis (*). B, Note the descending course and a small local collateral (arrow) bridging the stenosis $(*)$ in MPR of DynaCT datasets in volume rendering technique (VRT) from the same 3DRA study.

In one case with an arteriovenous malformation, the circumferential and perforating brain stem arteries were clearly hypertrophic as they were part of the AVM-feeding vessels. In comparison to the other cases, the quantity of vessels was also considerably higher and they could be displayed more clearly (Fig 5).

\section{DISCUSSION}

Current neuroradiologic angiographic equipment is able to detect the main anatomic features of small pontine arteries. Volume rendering and $\mathrm{CT}$-like reconstructions of $3 \mathrm{D}$ rotational datasets are superior to conventional DSA. A larger number of projections, like the subtraction technique with individual adjustment of threshold levels of flat panel CT and 3D reconstructions, increase the possibilities of image processing. In particular, transverse and parasagittal sections of volume rendering datasets with a section thickness between 5 to $10 \mathrm{~mm}$ include larger parts of the curved and descending course of the circumferential arteries. The visualization of the smaller direct pontine perforators demanded thinner sections around $3 \mathrm{~mm}$.

MPR with thick sections displayed the best results of the basic anatomic patterns of the small side branches of the basilar artery because of the general orientation of these penetrating vessels with their characteristic downward-curving route toward the pontine tegmentum. The con of using thicker reconstructed sections is a decline of spatial resolution in small vessel angiography. Some of the side branches cannot be displayed free from superimpositions, and the correct allocation to the left or right side is difficult.

Compared with the single frames of the $2 \mathrm{k}$ DSA series, the continuous acquisition of the 3D rotational angiography runs with a scan time of 5 seconds provides more reliable vessel detection (Figs 2 and 5). In 3D reconstructions, the small branches can be visualized free from superimpositions because of the increased number of projections and the longer acquisition time during pulsative vessel filling of these small branches.

Contrary to what might be expected from consideration of the total image acquisition count only, the patient radiation dose (reported in literature before) for a $3 \mathrm{D}$ rotational angiography acquisition is lower than for a biplanar DSA series. ${ }^{11}$

Venous contamination was widely excluded with early start and short scan durations of the scans and visual proof that small arteries originate directly from the basilar artery.

Imaging findings are coincident with previous anatomic descriptions based on examinations of injection specimens. ${ }^{8,9}$ However, compared with the anatomic literature, angiographic detectability of the small vessels of the basilar artery seems to be incomplete. With existing angiography techniques and reconstruction tools, it is not possible to display all circumferential 

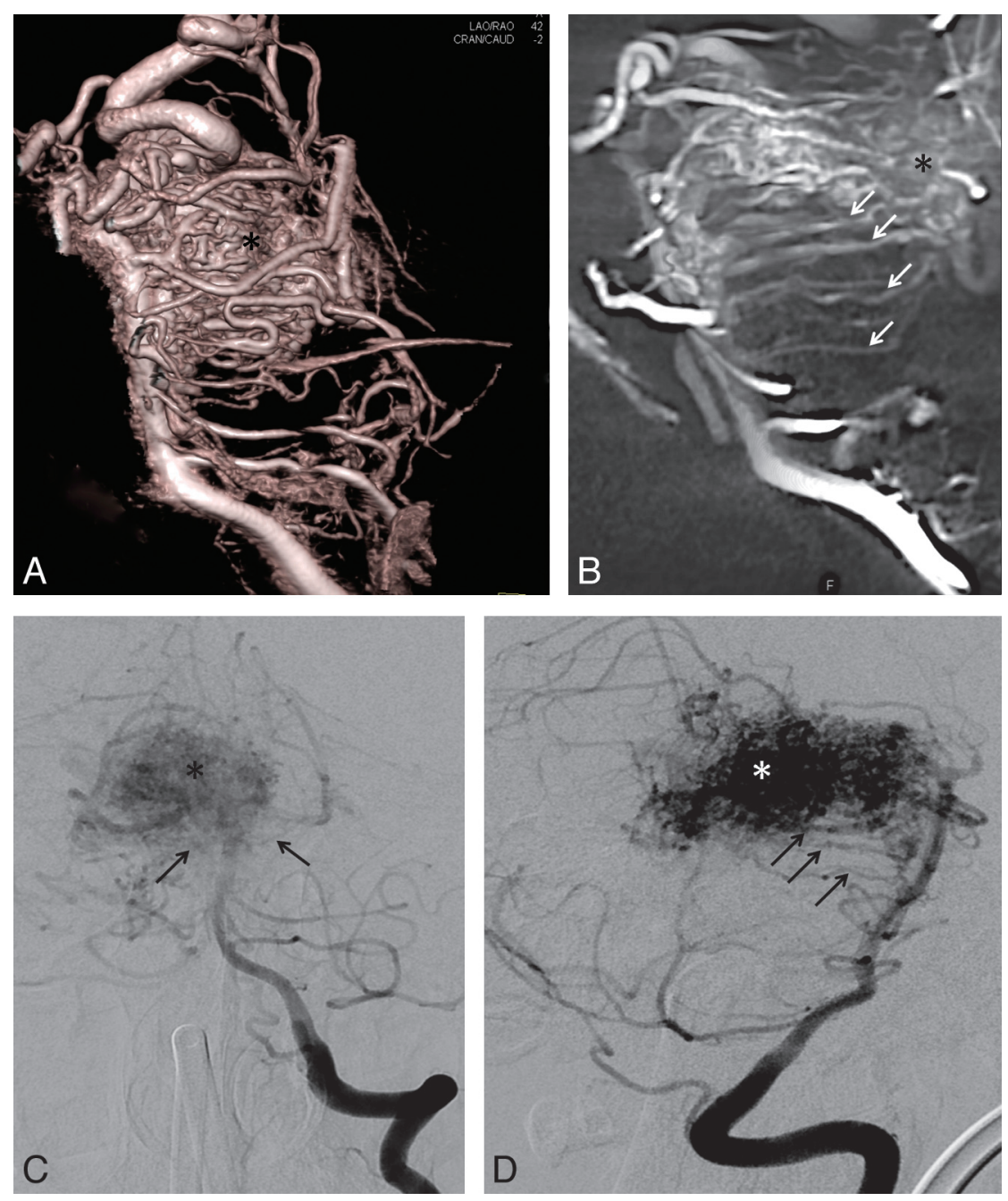

FIG 5. Volume rendering reconstruction $(A)$ and parasaggital cut of a DynaCT dataset $(B)$ show multiple hypertrophied circumferential pontine arteries (arrows) involved as feeders in the supply of a mesencephalic $\mathrm{AVM}\left({ }^{*}\right)$. Compared with the volume rendering reconstructions $A$ and $B, 2 \mathrm{k}$ DSA images of the same patient in standard projections, $C$ and $D$, show fewer circumferential arteries (arrows) and those that are displayed are less well visualized.

arteries and anastomoses for an exact anatomic definition of this region as found in descriptions based on anatomic preparations.

In addition, our results give rise to the assumption that there are variations with deviations from the common segmental pattern: in several cases, we observed irregular settings with common trunks and anastomoses between adjacent branches resembling a pial network similar to findings at the spinal cord (Fig 2). Due to limitations in spatial resolution, anastomoses as potential pathways for local collaterals are only proved in occasional cases (Fig 2). In the presence of a basilar or vertebral artery stenosis, only 1 small collateral could be detected in each of the 2 cases (Fig 4). More circumferential arteries and anastomoses were seen in an AVM case with hypertrophy of arterial feeders (Fig 5).

Similar to the sulco-comissural arteries in the spinal cord, which enter the spinal cord only in greater intervals, we could regularly detect 3 groups of direct pontine perforators extending from the dorsal surface of the basilar artery entering the brain stem at the ventral surface (Fig 3).

The practical value of our findings is unknown at the moment. However, in the context of endovascular interventions, it be- comes clear that almost no segment of the basilar artery is free from important side branches and display of these small vessels may contribute for risk assessment before angioplasty and stent placement. In the SAMMPRIS trial, most ischemic strokes after angiographically successful angioplasty and stent placement in the basilar artery in perforator territory. ${ }^{12}$ In addition, perforator infarcts after treatment of fusiform aneurysms of the basilar artery raise doubts about sufficient consideration of vascular anatomy. In conclusion, efforts at reducing complications from endovascular treatments of aneurysm, infarctions, or stenosis with intracranial angioplasty and stent placement should focus on reducing the risks of regional perforator infarction. ${ }^{12}$

For the sake of completeness, some basilar artery pathologies have no important branch vessels in the involved segment, eg, sidewall aneurysms between the origins of the circumferential pontine arteries or aneurysms arising from the ventral surface of the basilar artery, which is free from side branches.

\section{Limitations}

Variation between the cases may be attributed to anatomic variants or a lack of detectability of the small vessels. Incomplete or delayed filling with contrast material and limitation in the resolution of smaller and terminal branches may be reasons for the incomplete display of anatomic features. ${ }^{9,13,14}$ There are only a few descriptions of pontine arteries in the existing angiographic literature. ${ }^{7}$ Only with the use of high-field MR imaging were Kang et $\mathrm{al}^{15}$ able to detect similar patterns.

Incomplete contrast filling of the small branches in $2 \mathrm{k}$ DSA images depends on hand injection. Although details of technical safeguards for neuroangiography are seldom discussed in existing literature and contrast application via injector seems to be the standard in many institutes performing neuroangiography, in our opinion, hand injection in 2D DSA is safer and therefore the standard in our institute. ${ }^{16-18}$ Contrast application via injector was only selected for 3D DSA. The caliber of the vertebral arteries varies quite often and hand injection may avoid complications, for example, dissection or vessel rupture.

\section{CONCLUSIONS}

Our study proves the concept that modern neuroangiographic imaging with 3DRA and flat detector CT reconstructions is able to display small pontine arteries with patterns described in the anatomic literature.

Potential furture developments should focus on a continuous improvement of image quality and further refinement of techno- 
logical advances or concepts to increase reliability of small vessel angiography. Further development and improvement of applications are necessary. Limited spatial and contrast resolution of flat panel angiography as well as suboptimal protocols for injection of contrast materials are the main limitations of our approach. Possible avenues for future improvements should address the development of flat detectors with even higher resolution and faster and more flexible detector readout, allowing for faster scanning to improve image quality of small vessel angiograms. ${ }^{4}$

Remarkably, our findings were generated from historical datasets using standard clinical protocols. Dedicated angiographic protocols with optimization of scan time, iodine concentration, and injection parameters of contrast materials may provide future options to use this data for risk assessment of patients with arterial occlusive or stenotic disease or aneurysms before endovascular treatment with angioplasty and stent placement.

This was a proof of concept study about the feasibility to visualize the small side branches of the basilar artery by use of modern angiographic equipment and reconstructions tools. The sample size of this study is too low for an exact anatomic definition of this anatomic region. Currently, further research on small vessel angiography will be necessary and should address technical optimization to improve image quality as well as clinical validation in larger studies to become a routine procedure in neuroangiography. Future studies should be evaluated blinded to avoid exaggeration of the results and should compare different angiographic techniques.

Disclosures: Stephanie Lescher received support for travel to the RSNA 2013 meeting from Siemens Health Care AG, related to the study. Joachim Berkefeld received a consulting fee or honorarium* and travel support* related to the study; the project is part of a scientific cooperation between Siemens and the Institute of Neuroradiology at the University Frankfurt, Germany, and travel expenses for presentation of the project are covered by Siemens. *Money paid to institution.

\section{REFERENCES}

1. Piotin M, Blanc R, Kothimbakam R, et al. Primary basilar artery stenting. AJR Am J Roentgenol 2000;175:1367-69
2. Chimowitz MI, Lynn MJ, Derdeyn CP, et al. Stenting versus aggressive medical therapy for intracranial arterial stenosis. $N$ Engl J Med 2011;365:993-1003

3. Lang J. Clinical Anatomy of the Posterior Cranial Fossa and its Foramina. Stuttgart: Thieme; 1991

4. Kalender WA, Kyriakou Y. Flat-detector computed tomography (FD-CT). Eur Radiol 2007;17:2767-79

5. Mercier PH, Brassier G, Fournier HD, et al. Vascular microanatomy of the pontomedullary junction, posterior inferior cerebellar arteries, and the lateral spinal arteries. Interv Neuroradiol 2008;14:49-58

6. Mercado R. Santos-Franco J, Ortiz-Velazquez I, et al. Vascular anatomy of the foramen of Vicq d'Azyr: a microsurgical perspective. Minim Invasive Neurosurg 2004;47:102-06

7. Lasjaunias P, Berenstein A, Ter Brugge KG. Surgical Neuroangiography. Berlin-Heidelberg: Springer-Verlag; 2001

8. Marinkovic $S$, Gibo $H$. The surgical anatomy of the perforating branches of the basilar artery. Neurosurgery 1993;33:80-87

9. Duvernoy HM. Human Brain Stem Vessels. Berlin Heidelberg: Springer-Verlag; 2010

10. Kyriakou Y, Struffert T, Dörfler A, et al. Basic principles of flat detector computed tomography (FD-CT). Radiologe 2009;49:811-19

11. Schueler B, Kallmes D, Cloft H. 3D cerebral angiography: radiation dose comparison with digital subtraction angiography. AJNR Am J Neuroradiol 2005;25:1898-901

12. Derdeyn CP, Fiorella D, Lynn MJ, et al. Mechanisms of stroke after intracranial angioplasty and stenting in the SAMMPRIS trial. Neurosurgery 2013;72:777-95

13. Pai BS, Varma RG, Kulkarni RN, et al. Microsurgical anatomy of the posterior circulation. Neurol India 2007;55:31-41

14. Pais D, Arantes M, Casal D, et al. Brain stem arteries in Canis familiaris-implications in experimental procedures. Braz J Morphol Sci 2009;26:8-11

15. Kang CK, Park CA, Kim KN, et al. Non-invasive visualization of basilar artery perforators with 7T MR angiography. JMRI 2010; 32:544-50

16. Yousem DM, Trinh BC. Injection rates for neuroangiography: results of a survey. AJNR Am J Neuroradiol 2001;22:1838-40

17. Fox AJ. Technical aspects of neuroangiography: are risks and safeguards understood in the same way? AJNR Am J Neuroradiol 2001;22:1809-10

18. Gailloud $P$, Murphy K. Risk of cerebral angiographic complications, injection volumes, and rates. AJNR Am J Neuroradiol 2002;23:893; author reply 893-94 